Cahiers

Balkaniques

\section{Cahiers balkaniques}

Hors-série | 2015

Les élites grecques modernes, $\mathrm{XVIII} \mathrm{e}^{\mathrm{X}} \mathrm{XX}^{\mathrm{e}}$ siècles :

identités, modes d'action, représentations

\title{
Aristophane sur la scène grecque moderne
}

Un théâtre populaire ou un théâtre fait par et pour les happy-few ?

Aristophanes on the Modern Greek Stage: a Popular Theatre or a Theatre made

by and for the 'Happy Few'?

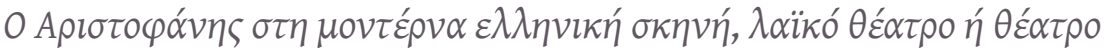

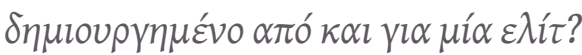

\section{Kaiti Diamantakou-Agathou}

\section{OpenEdition \\ Journals}

Édition électronique

URL : https://journals.openedition.org/ceb/5533

DOI : 10.4000/ceb.5533

ISSN : 2261-4184

Éditeur

INALCO

\section{Édition imprimée}

Date de publication : 1 mars 2015

ISBN : 978-2-85831-224-5

ISSN : 0290-7402

\section{Référence électronique}

Kaiti Diamantakou-Agathou, « Aristophane sur la scène grecque moderne », Cahiers balkaniques [En ligne], Hors-série | 2015, mis en ligne le 10 décembre 2015, consulté le 06 juillet 2021. URL : http:// journals.openedition.org/ceb/5533; DOI : https://doi.org/10.4000/ceb.5533

Ce document a été généré automatiquement le 6 juillet 2021. 


\section{Aristophane sur la scène grecque moderne}

Un théâtre populaire ou un théâtre fait par et pour les happy-few ?

Aristophanes on the Modern Greek Stage: a Popular Theatre or a Theatre made

by and for the 'Happy Few'?

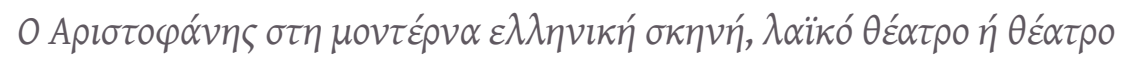

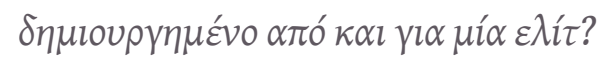

Kaiti Diamantakou-Agathou

\section{La comédie antique, ses origines populaires et son évolution civique}

1 Commençons par la reprise d'informations bien connues et banales. Liée dès sa genèse à la démocratie (ARISTOTE, Poét. 3, 2-3, 1448a), la comédie antique, issue de rituels phalliques et des cortèges dionysiaques (ibidem 4, 10-12, 1449a), devint - après une évolution en plusieurs étapes - un genre dramatique distinct, ayant son propre code esthétique et son propre statut socioculturel, comme on peut les étudier, par excellence, dans les onze comédies d'Aristophane du ve siècle et du début du IV siècle av. J.-C. En d'autres termes, la comédie antique fut une sorte de théâtre indéniablement populaire du point de vue de ses origines et, en même temps, civique du point de vue de son insertion dans le milieu social de son époque. À l'instar du Carnaval, la comédie aristophanesque possédait une fonction de compensation sociale et de mise en question à caractère ludique : mise en question de la politique et de son exercice abusif, des autorités de la cité, des dieux et de leurs serviteurs terrestres, des savants, des poètes, des citoyens anonymes ou éponymes, de toute sorte de métier, de sexe, d'âge, de culture, d'orientation politique ou sexuelle. Cela dit, au contraire du Carnaval, les comédies d'Aristophane étaient présentées dans le cadre officiel des Grandes Dionysies ou des Lénéennes, toujours sur le site consacré du Théâtre de Dionysos (THIERCY, 1986, 24), devant les autorités de l'État-cité, ainsi que devant une 
grande partie du corps civique des Athéniens, dont la majorité, on peut le supposer, se composait des habitants du centre-ville d'Athènes. Le caractère «bourgeois » de cette œuvre se reflète, entre autres, dans les lieux dramatiques inscrits dans les textes qui ont survécu, lieux qui, dans la plupart des cas, sont des sites réels de l'Athènes antique. La satire politique et sociale se déroule tantôt dans le Pnyx, tantôt devant les Propylées, tantôt devant (ou dans) le Lieu de Réflexion de Socrate, tantôt sur le lieu sacré du Festival des Thesmophories, tantôt devant des maisons urbaines de citoyens athéniens... tandis que, même lorsque l'action se déplace en des lieux imaginaires (le domicile des Dieux dans le ciel, les Enfers, le royaume des Oiseaux dans l'air), la carte urbaine athénienne reste toujours vibrante et omniprésente au niveau verbal et narratif (KONSTAN, 1997, 3-17).

2 Le monde d'Aristophane constitue, donc, un monde rituel littérarisé et, de ce fait, un monde populaire urbanisé, dans la production et la perception duquel coexistent le "goût de la réflexion " et le "goût des sens", le "goût pur " et le "goût impur", l'« imagination populaire » et la « sociodicée esthétique », comme dirait Pierre BOURDIEU (1979, 569-574). Il s'agit d'un amalgame unique qui impliquait toute la ville-cité et ses habitants, mais dont les spectateurs idéaux et visés étaient en premier lieu les détenteurs du privilège d'être "Athéniens-libres-hommes-adultes-cultivés ", à savoir ceux qui fréquentaient le théâtre de Dionysos sur le versant sud-ouest de l'Acropole et qui, en même temps, déterminaient, - par leur participation régulière aux différentes institutions constitutives du régime athénien -, la vie politique, financière, judiciaire, sociale et culturelle de leur ville-cité.

\section{Aristophane et sa réception par la scène grecque moderne : les trois restaurateurs}

3 Ce type de théâtre polyphonique, au niveau autant de la forme que du contenu, disparut en pratique en même temps que la démocratie directe athénienne ; il survécut par la suite pendant des siècles uniquement en tant que monument textuel, pour ne ressusciter théâtralement dans son pays natal qu'à la fin du XIX ${ }^{e}$ siècle ${ }^{1}$ et se propager par la suite (et s'altérer) pendant les premières décennies $\mathrm{du} \mathrm{xx}^{\mathrm{e}}$ siècle, sous forme de spectacles quasi pornographiques, destinés à des publics exclusivement masculins et privés de toute ambition d'explorer largement et à fond le tissu compliqué des textes d'Aristophane. Depuis les années 1930 du siècle précédent jusqu'au début de ce nouveau millénaire, l'investigation et la restitution esthétique et idéologique d'Aristophane doivent beaucoup à trois metteurs en scène, sur la contribution desquels on va centrer notre intérêt dorénavant. Il s'agit de trois metteurs en scène qui se sont occupés de la comédie antique d'une façon continue, cohérente, claire et distincte, souvent en dialogue (ou en confrontation) bilatérale directe entre eux, et dont les différentes " écoles " servent jusqu'à nos jours de points de référence (affirmative ou négative) tant pour les praticiens que pour les théoriciens et les historiens du théâtre, en Grèce et à l'étranger : Karolos Koun, Alexis Solomos et Spyros Evangelatos. Voici très brièvement leur portrait biographique :

Karolos Koun, né à Prousa (1908) et mort à Athènes (1987), était fils d'un commerçant cosmopolite, Errikos Koen, à moitié grec-orthodoxe et à moitié allemand-polono-juif. Élevé à Constantinople dans une maison bourgeoise, entouré de plusieurs précepteurs, 
il acheva ses études secondaires à l'École américaine réputée de Constantinople, le Robert College. En 1928 il suivit des cours d'esthétique à la Sorbonne, pour s'installer définitivement à Athènes en 1929 et travailler, de 1930 à 1938, en tant que professeur de langue et de lettres anglaises au Collège Américain, où il entreprit, avec ses jeunes étudiants, ses premières représentations. En 1934, il crée avec Yannis Tsarouchis et Dionysis Devaris la Scène Populaire, qui sera dissoute en 1936 pour des raisons économiques. Après cette dissolution et son départ du Collège Américain, Karolos Koun coopère en tant que directeur jusqu'en 1941 avec les troupes des actrices fameuses de l'époque, Katerina Andreadi et Marika Kotopouli. En 1942, au cœur de l'Occupation allemande et dans des conditions économiques et de survie très difficiles, Koun crée le Théâtre d'Art, qui, après une période très dense en production artistique, en problèmes financiers et en oscillations interpersonnelles, sera obligé de s'interrompre, d'abord en 1945, puis en 1949. En 1954, le Théâtre d'Art recommence à fonctionner, dorénavant logé en permanence à l'amphithéâtre en sous-sol de la Galerie Orphée, pour suivre désormais une route pleine de succès et de signes de reconnaissance : lauréat pour les Oiseaux au Festival des Nations à Paris en 1962, plusieurs invitations faites à Karolos Koun de diriger au sein des plus grands théâtres d'Europe et d'Amérique, plusieurs invitations adressées au Théâtre d'Art pour participer à des Festivals en Grèce et à l'étranger, participation continuelle du Théâtre d'Art au Festival d'Épidaure après la chute de la junte et la fin du monopole du Festival accordé au Théâtre national de Grèce, attribution à Karolos Koun de la Médaille Phœnix par l'État grec et de la Médaille d'Argent par l'Académie d'Athènes, concession honorifique par l'État grec d'un deuxième espace pour le théâtre, au centre d'Athènes, où loge jusqu'à nos jours la Deuxième Scène du Théâtre d'Art (MAVRomoustaKos, 2008, 17-41).

5 Alexis Solomos (1918-2012), après avoir accompli ses études primaires et secondaires au Collège Américain d'Athènes, où il fut élève de Karolos Koun, et après avoir commencé des études à la Faculté de Droit d'Athènes, suivit une formation théâtrale exigeante, d'abord à l'École Dramatique du Théâtre national de Grèce (1939-1942), puis à l'Académie Royale de Londres (1945-1946), à l'Université de Yale aux États-Unis et à l'Atelier Dramatique d'Erwin Piscator à New York (1946-1948); cela fut entrecoupé d'intervalles professionnels en Grèce, parmi lesquels on note sa coopération en tant qu'acteur en 1943 avec le Théâtre d'Art, où fut montée l'année suivante sa pièce de jeunesse, le Dernier Blanc-corbeau. Quelques années plus tard, Alexis Solomos débute sa carrière de metteur en scène à New York (au Cherry Lane Theatre et au Province Town Playhouse, 1947) et puis à Londres (Embassy Theatre, 1949), pour revenir par la suite en Grèce et amorcer une carrière féconde jusqu'en 1992, lorsqu'il se retire définitivement du théâtre. Il a coopéré avec le Théâtre national de Grèce, le Théâtre national de Grèce du Nord, la Scène lyrique nationale de Grèce et plusieurs autres troupes théâtrales; il fonda aussi sa propre troupe théâtrale, nommée Proskénion (1967-1972 et occasionnellement après 1978). Il occupa également le poste d'administrateur adjoint de l'Établissement national de la Radiodiffusion (1974-1975), ainsi que celui d'administrateur du Théâtre national de Grèce (à deux reprises : 1980-1983 et 1990). Outre ses nombreuses mises en scène, son apport d'auteur fut également vaste et riche : des pièces théâtrales, plusieurs études, - souvent rééditées -, sur divers champs de l'histoire du théâtre, plusieurs traductions de pièces de théâtre - parmi lesquelles, les Oiseaux et les Thesmophories d'Aristophane, publiées sous le pseudonyme de A. Rosolymos. Pour l'ensemble de son apport aux Lettres et aux Arts, l'État grec lui a attribué la Médaille du Phœnix (degré de Commandeur), l'Académie d'Athènes l'a 
également honoré et le Département d'études théâtrales de l'Université d'Athènes l'a nommé au grade de docteur honoraire en 1997 (solomos, 1980, et passim)2 .

Beaucoup plus jeune, Spyros Evangelatos (1940-), originaire d'une famille d'artistes renommés (père compositeur, mère harpiste), a fait des études à la Faculté de Philosophie d'Athènes où il a reçu le titre de docteur es Lettres en 1970. Il a aussi fait des études de théâtre à l'École Dramatique du Théâtre national de Grèce et à l'Université de Vienne, en suivant des cours et des séminaires théâtraux dans plusieurs pays d'Europe. Depuis sa jeunesse jusqu'à nos jours, Spyros Evangelatos a réussi à combiner et à développer sa formation académique et scientifique en voie parallèle et complémentaire de son travail artistique : d'une part, philologue, écrivain, professeur des Universités, membre régulier, plus tard vice-président et président actuel de l'Académie de Grèce ; d'autre part, directeur inlassable, au sein de plusieurs organismes théâtraux, grecs et étrangers; fondateur de la Scène Néohellénique (1962), à laquelle succéda l'Amphithéâtre, qui n'a cessé d'alimenter le paysage théâtral de 1975 jusqu'en 2011, lorsqu'il fut obligé de suspendre ses fonctions pour des raisons économiques ; directeur général artistique du Théâtre de Grèce du Nord (1977-1980) ainsi que de la Scène lyrique nationale (1997-1999). Enfin, pour ne mentionner que certaines des distinctions honorifiques qu'il a reçues : prix « Karolos Koun » (1988), prix de la Société des auteurs dramatiques Grecs (1994), titre de "Cavaliere Ufficiale", décerné par le Gouvernement italien (1995), prix « Fotos Politis », décerné par le Musée et le Centre d'étude du théâtre grec (1997), titre de «commandeur de l'Ordre du Phénix» (2000), attribué par le président de la République grecque (viviLAKIS, 2001, 19-20).

\section{Approches et divergences}

7 Dotés de ressources culturelles importantes et institutionnalisées, les trois metteurs en scène en question ont réussi progressivement, d'une façon systématique et cohérente, tout au long de leur carrière artistique, à acquérir de surcroît un capital social important, lié à la possession d'un réseau durable de relations d'interconnaissance et d'inter-reconnaissance; ils ont donc réussi à augmenter et à consolider leur pouvoir symbolique auprès de la société grecque moderne. Et dans cette consolidation de leur statut symbolique, leur attachement à tous trois au service de la comédie antique joua un rôle décisif: Karolos Koun dirigea d'abord au sein du Collège Américain d'Athènes, puis au sein de la Scène Populaire et surtout au sein du Théâtre d'Art sept des onze comédies d'Aristophane, certaines à plusieurs reprises, tandis que son disciple, Giorgos Lazanis, assuma la mise en scène de deux autres comédies, les Cavaliers (1979) et les Guêpes (1981), toujours au sein du Théâtre d'Art, et avant la mort de son maître ${ }^{3}$; Alexis Solomos dirigea au sein du Théâtre national de Grèce de 1956 à 1980 et occasionnellement au sein du Proskénion dix des onze comédies survivantes, parmi lesquelles les "pièces féminines" à plusieurs reprises ${ }^{4}$; enfin, la contribution de Spyros Evangelatos, en commençant par le Théâtre de Grèce du Nord en 1969 et en continuant avec l'Amphithéâtre de 1976 jusqu'en 2006, s'élève à neuf productions différentes de sept comédies d'Aristophane, Lysistrata et l'Assemblée des femmes étant les deux comédies qu'il a révisées ${ }^{5}$. L'inter-concurrence ou l'inter-complémentarité entre ces trois metteurs en scène est visiblement attestée par certains choix du répertoire : Alexis Solomos ne monta jamais Ploutos qui avait été une des mises en scène de départ 
de Karolos Koun au Collège Américain et au sein de la Scène Populaire, tandis qu'il s'occupa des Oiseaux seulement en 1979, vingt ans après la représentation légendaire de cette comédie par le Théâtre d'Art à l'Odéon d'Hérode Atticus en 1959. Karolos Koun ne travailla jamais l'Assemblée des femmes, qui fut la première comédie dirigée par Alexis Solomos ; enfin, Spyros Evangelatos, de son côté, choisit au début de se pencher sur les comédies préférées de ses prédécesseurs, d'abord l'Assemblée des femmes et Lysistrata, les deux premières mises en scène d'Alexis Solomos, puis les Grenouilles et le Ploutos, les premières mises en scène d'Aristophane par Karolos Koun.

8 Loin de se limiter à des questions de répertoire, la relation - qu'elle soit d'accord ou de contestation -, entre ces trois metteurs en scène, fut beaucoup plus profonde du point de vue esthétique et idéologique. Le signal de départ fut donné par Karolos Koun, qui dans les années 1930, commence à former et à proposer son code d'approche à la comédie antique dans le cadre scolaire, - et profondément bourgeois - du Collège Américain, ainsi que dans le cadre mi-amateur mi-professionnel de la Scène Populaire (de courte durée, 1934-1936). Sur les fondements de ces premières représentations de laboratoire le Théâtre d'Art érigera son approche systématique de la comédie antique depuis les années 1950 et jusqu'aux années 1980, approche marquée par une distanciation progressive face au réalisme imitatif pour aller vers l'« expressionnisme populaire ». C'est ainsi que Karolos Koun lui-même qualifiait sa propre esthétique, c'est-à-dire l'esthétique d'une vérité primitive et subjective, nourrie par des symboles issus de la tradition populaire et socioculturelle de toutes les périodes de l'hellénisme, comme elle fut comprise et formée à travers les filtres bourgeois du metteur en scène (GLYTZOURIS, 2001, 591-595). En confrontation constante - plutôt « construite » et non pas essentielle -, avec le discours dominant du Théâtre national, Karolos Koun a puisé des éléments autant dans le réservoir de l'avant-garde moderniste occidentale que dans la tradition populaire grecque, pleine d'influences orientales (théâtre d'ombres, carnaval, diverses performances parathéâtrales), en équilibrant efficacement la vraisemblance stylisée et l'abstraction complète, la fonction carnavalesque et la fonction didactique de la comédie antique (MAVROMOUSTAKOS, 2008, 27-29).

9 En suivant une voie tout à fait parallèle aux représentations professionnelles de Karolos Koun, depuis 1956 (représentation de l'Assemblée des femmes, un an avant le Ploutos du Théâtre d'Art) jusqu'en 1986 (répétition de Lysistrata par le Proskénion un an après les Thesmophories du Théâtre d'Art et un an avant la mort de Karolos Koun), Alexis Solomos introduisit Aristophane au Festival d'Épidaure et fut le porte-parole solide et irremplaçable de la politique culturelle nationale à ce sujet, suivant l'idéologie dominante d'européanisation de la période d'après-guerre, une idéologie qui dépendait de et était déterminée par les valeurs et les besoins esthétiques d'une société par excellence bourgeoise, qui nourrissait et supportait le festival et les autres manifestations mondaines de l'époque. Au moyen, en premier lieu, des traductions "décentes » de Thrasyvoulos Stavrou, qui étaient épurées de toute obscénité, et en empruntant des éléments variés à différents réservoirs dramatiques (boulevard, farce, opérette, revue grecque, cirque), Alexis Solomos proposa une version élégante et "correcte", spectaculaire et divertissante et, de ce fait, absolument capable de propager un Aristophane inoffensif et anodin et de le consacrer en tant que «capital national ", propice à être mis en valeur touristique autant que la tragédie antique (vAN STEEN, 2000, 197-204). 
10 Une synthèse féconde entre la thèse "moderniste ", "juteuse ", «bruyante ", "jubilatoire», "populaire» de Karolos Koun et l'antithèse "modernisante», "élégante ", " décente", "européanisée » d'Alexis Solomos", est entreprise par Spyros Evangelatos qui se mit à l'agôn théâtral comique en 1969 et y resta jusqu'en 2006. En somme, à Spyros Evangelatos on doit une approche éclectique et syncrétique du point de vue esthétique, nuancée selon les conditions formelles et sémantiques spécifiques de chaque comédie, fondée sur une lecture philologique profonde et servie par une grande variété d'acteurs, venus des écoles dramatiques différentes. La tendance à l'adaptation partielle de l'original par des additions, des retranchements ou des transformations de certaines séquences, les interventions métathéâtrales du metteur en scène en tant que narrateur brechtien entre les actes ou à la fin d'une pièce, la mise en valeur de la dialectique aristophanesque et de l'ambiguïté profonde sous la surface textuelle jubilatoire surtout dans la partie finale de ses représentations, last but not least, la mise en exergue du caractère urbain de certaines pièces d'Aristophane et leur transposition dans des cadres scéniques bourgeois actualisés, sont quelques offrandes représentatives de Spyros Evangelatos sur l'autel de la comédie antique qui, grâce à lui, sera éclairée par des nuances sémantiques subtiles, souvent en faveur d'un rire significatif et réfléchi et au détriment du rire spontané et absolu (MAVROGENI, 2006, 289-298).

\section{Aristophane par qui et pour qui ?}

11 Pas toujours happy, voire pas du tout happy pendant certaines périodes de leur vie, ils étaient sûrement few ceux qui se sont voués à la restitution théatrale et la (re)diffusion culturelle de la comédie antique. À travers leur œuvre artistique et leur activité de grande envergure socioculturelle, Aristophane a été consacré définitivement sur la scène grecque moderne en tant que "valeur sûre de l'héritage, [qu'] il n'est plus convenable de mettre en question: elle est nécessairement profitable à qui la consomme.» (PAVIS, 1990, 54); inversement, à travers notamment - entre autres auteurs dramatiques - Aristophane et son "effet classique" (idem, 54-56), ces trois metteurs en scène se sont, à leur tour, consacrés sur la scène grecque moderne, en tant que serviteurs d'« un théâtre à objet culturel, en contribuant par la qualité de leur travail à la formation du spectateur dans une optique éthique et/ou esthétique plus ou moins marquée » (GOUDON, 1982, 11).

Pendant cet échange de services culturels et cette réinsertion de poids symbolique, sur une période d'un demi-siècle à peu près, dans une Grèce en voie d'européanisation croissante, quel a pu être le profil et le profit des spectateurs grecs modernes, à qui ont été livrées, comme un héritage public, les comédies d'Aristophane? Deux recherches qui ont eu lieu en Grèce, une première menée par la revue Highlights (KAPODISTRIA \& KYRGIA, 2007, 583-593) et une deuxième, menée dans le cadre du Centre National du Théâtre et de la Danse, sous la direction du sociologue Nikos Panayotopoulos (2012), n'ont pas inclus le théâtre d'Épidaure, parmi les quinze ou les neuf, respectivement, espaces de théâtre échantillonnés, et la comédie antique n'y constituait pas une catégorie spécifique, ce qui nous ôte des données concrètes sur sa réception. Cependant, on pourrait supposer que les résultats statistiques - s'ils existaient - sur la composition du public d'Aristophane à la manière de Koun, de Solomos ou d'Evangelatos, ne se différencieraient peut-être pas beaucoup des résultats généraux sur le profil du public 
théâtral grec contemporain. Or ces résultats montrent que les pratiques et les préférences de chaque spectateur dépendent, en principe, de la structure de son héritage, c'est-à-dire de son capital économique et culturel, ainsi que de son itinéraire social qui oriente et détermine la relation qu'il entretient avec cet héritage (PANAYOTOPOULOS \& VIDALI, 2012, 92).

On se contente, donc, de s'interroger pour savoir si dans le cas du théâtre d'Aristophane, dont le "maître mot est la contestation " (MARÉCHAUX, 1996, 34), se retrouve également la constatation de Pierre Bourdieu quant à la fréquentation des musées et au public qui en tire profit : il s'agit d'« une libéralité factice, puisque l'entrée libre [N.B. payée dans le cas du théâtre] est aussi entrée facultative, réservée à ceux qui, dotés de la faculté de s'approprier les œuvres, ont le privilège d'user de cette liberté et qui se trouvent par là légitimés dans leur privilège, c'est-à-dire dans la propriété des moyens de s'approprier les biens culturels ou, pour parler comme Max Weber, dans le monopole de la manipulation des biens de culture et des signes institutionnels du salut culturel » (BOURDIEU, 1969, 166-167).

\section{BIBLIOGRAPHIE}

BOURDIEU Pierre, 1979, la Distinction. Critique sociale du jugement, Paris : Les Éditions de Minuit.

BOURDIEU Pierre, 1996, l'Amour de l'art. Les musées d'art européens et leur public, Paris : Les Éditions de Minuit.

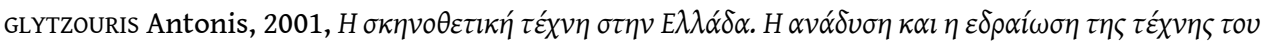

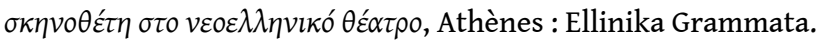

GOUDRON Anne-Marie, 1982, Théâtre, Public, Perception, Paris : Éditions du CNRS.

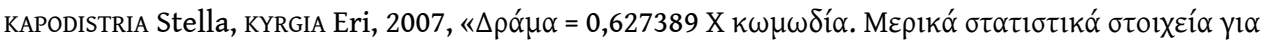

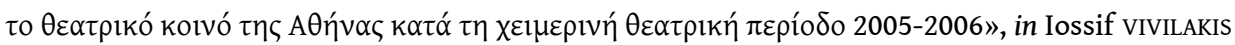

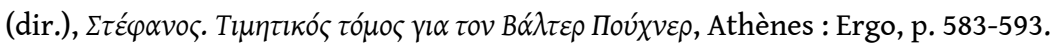

KONSTAN David, 1997, “The Greek Polis and Its Negations. Versions of Utopia in Aristophanes' Birds”, in Gregory W. DOBRov (dir.), The City as Comedy, Society and Representation in Athenian Drama, North Carolina: The University of North Carolina Press, pp. 3-22.

MARÉCHAUX Pierre, 1996, « le Théâtre antique », in Dominique BERTRAND et alii, le Théâtre, Paris : Bréal, p. 12-68.

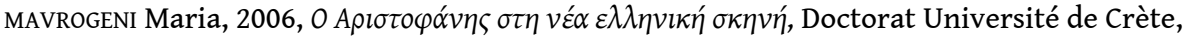
Département de Philologie.

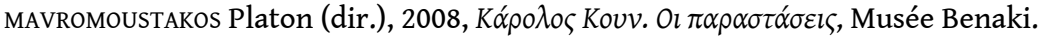

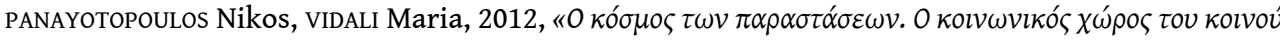
$\tau \omega \nu \theta \varepsilon \alpha \dot{\tau} \tau \omega \nu »$. Koinonikes Epistimes/Sciences Sociales, vol. I, p. 64-94.

PAVIS Patrice, 1990, le Théâtre au croisement des cultures, Paris : José Corti. 


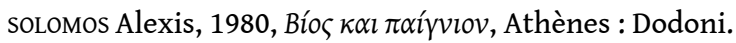

solomos Alexis, 1972, (1961 en grec), Aristophane vivant, (trad. J. Dalègre), Paris : Hachette.

THIERCY Pascal, 1986, Aristophane : fiction et dramaturgie, Paris : Les Belles Lettres.

VAN STEEN Gonda, 2000, Venom in Verse. Aristophanes in Modern Greece, Princeton: Princeton

University Press.

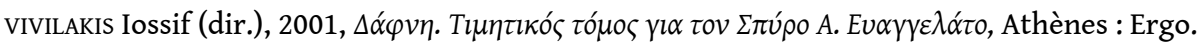

\section{NOTES}

1. À travers d'abord le Ploutos, la dernière comédie d'Aristophane, qui, selon l'« adaptation libre » de M. CHourmouzis (1861), fut montée par la troupe «Sophocle » de Sophocle Karydis en 1868 au "Théâtre d'Athènes", en constituant la première représentation professionnelle d'une comédie aristophanesque dans l'ère grecque moderne.

2. Voir aussi les discours de Spyros Evangelatos et de Walter Puchner sur l'apport théâtral (sur scène et sur papier) d'Alexis Solomos à l'occasion de sa nomination au grade de docteur honoraire par le Département d'études théâtrales de l'Université d'Athènes : http://www.theatre.uoa.gr/didaktiko-dynamiko/epitimoi-didaktores/ ale3hs-solomos.html, consulté le 28 octobre 2015.

3. Ploutos (1936, 1957), Grenouilles (1933, 1966), Lysistrata (1969), Paix (1977), Thesmophories (1985), Acharniens (1976, 1986), Oiseaux (1932, 1938, 1959, 1962, 1975).

4. Assemblée des femmes. (1956, 1981 : traduction par lui-même sous le pseudonyme de A. Rosolymos), Lysistrata (1957, 1958, 1960, 1980, 1986), Thesmophories (1958, 1978, 1979, 1982 : traduction de A. Rosolymos [voir A. Solomos]), Acharniens (1961), Guêpes (1963), Paix (1964), Cavaliers (1968, 1969, 1976), Nuées (1970), Grenouilles (1973).

5. Assemblée des femmes $(1969,1998)$, Lysistrata (1971, 1976), Grenouilles (1977), Ploutos (1978), Paix (1984), Nuées (1989), Guêpes (2006). Pour des données sur les représentations des comédies aristophanesques par le Théâtre national, le Théâtre d'Art et l'AmphiThéâtre voir maintenant la base de données "Aristophane sur la Scène Grecque Moderne" " [en grec] sur: http://users.uoa.gr/ diamcat/aristophanes/, consulté le 28 octobre 2015.

6. Ce n'est peut-être pas un hasard si, après la représentation de Lysistrata par Karolos Koun en 1969, où plusieurs rôles féminins avaient été joués par des acteurshommes, la Lysistrata de Evangelatos en 1976 a été jouée uniquement par des hommes, une convention que Karolos Koun adopta aussi, à son tour, dans ses Acharniens de la même année (1976), sa Paix (1977) et ses Thesmophories (1985). Et ce n'est peut-être pas un hasard également, si dans les programmes de l'Amphi-Théâtre on trouve très souvent des extraits des livres d'Alexis Solomos et surtout de son livre renommé et bien vivant jusqu'à maintenant, Aristophane vivant (1961, première édition). 


\section{RÉSUMÉS}

L'article centre son intérêt sur trois metteurs en scène grecs, qui ont par excellence façonné la réception grecque moderne de la Comédie Antique: Alexis Solomos, Karolos Koun et Spyros Evangelatos, représentants pareillement d'une élite culturelle et sociale, en faveur de laquelle ils ont exploré, d'une façon complémentairement créative et efficace, le théâtre soidisant « populaire » d'Aristophane.

The article focuses on three Greek directors, who have par excellence contributed -each one by his own esthetic and ideological means-, to the consecration and consolidation of Aristophanes' oeuvre on modern Greek stage: Karolos Koun, Alexis Solomos and Spyros Evangelatos, all of them representatives of a cultural and social elite, on whose benefit they explored, in a complementary way, the so-called "popular" theatre of Aristophanes.

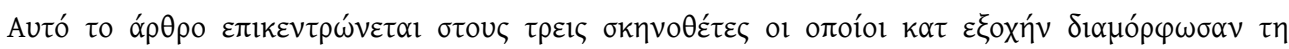

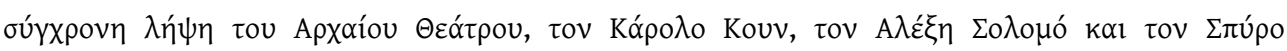

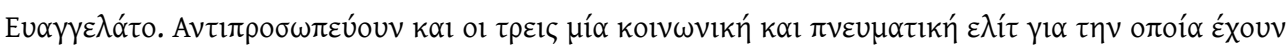

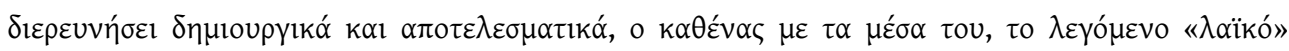

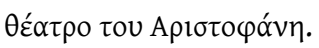

\section{INDEX}

\section{Index géographique : Grèce}

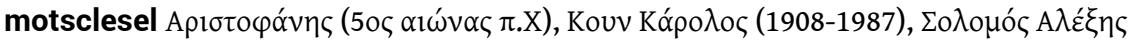

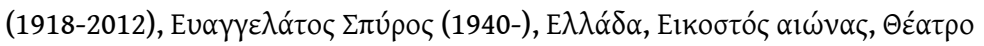

motsclestr Aristophanes (J.C. önce 5. yüzyıl), Koun Carolos (1908-1987), Solomos Alexis

(1918-2012), Evangelatos Spyros (1940-), Yunanistan, Yirminci yüzyıl, Tiyatro

motsclesmk АРИСТОФАН (5 ВЕК ПРЕД Ј.С.), КУН КАРОЛОС (1908-1987), СОЛОЧОС АЛЕХИС

(1918-2012), ЕВАГЕЛАТОС СПИРОС (1940-), ГРЦИЈА, ДБАЕСЕТТИОТ БЕК, ТЕАТАР

Thèmes : Théâtre

Keywords : Aristophanes (ve century BC.), Koun Carolos (1908-1987), Solomos Alexis (1918-2012), Evangelatos Spyros (1940-), Greece, Twentieth century, Theatre

Mots-clés : Aristophane (ve siècle av. J.-C. 445-375), Koun Carolos (1908-1987), Solomos Alexis

(1918-2012), Evangelatos Spyros (1940-)

Index chronologique : vingtième siècle

\section{AUTEUR}

\section{KAITI DIAMANTAKOU-AGATHOU}

Université d'Athènes 